\title{
CONSÓRCIO DE EXPORTAÇÃO DE SOFTWARE: PEQUENAS E MÉDIAS EMPRESAS SOB A PERSPECTIVA DE REDES SOCIAIS.
}

\author{
SOFTWARE EXPORT CONSORTIUM: SMALL AND MEDIUM \\ COMPANIES UNDER THE PERSPECTIVE OF SOCIAL NETWORKS.
}

\begin{abstract}
Ana Paula de Lacerda Zacharias ${ }^{1}$; Márcia Freire de Oliveira ${ }^{2}$; Mauro Rocha Côrtes ${ }^{3}$ ${ }^{1}$ Universidade Federal de São Carlos - UFSCar - São Carlos - Brasil anapaula@huc.com.br ${ }^{2}$ Universidade Federal de São Carlos - UFSCar - São Carlos - Brasil márciaf@dep.ufscar.br ${ }^{3}$ Universidade Federal de São Carlos - UFSCar - São Carlos - Brasil mauro@dep.ufscar.br
\end{abstract}

\begin{abstract}
Resumo
Este artigo tem como objetivo verificar se a organização de empresas em consórcios favorece a inserção exportadora de pequenas e médias empresas brasileiras do setor de software. A verificação é suportada pela perspectiva de redes sociais e a metodologia utilizada é a da pesquisa qualitativa, através de estudo de caso cujo objeto foi o Actminds, consórcio de exportação de software localizado em Campinas-SP. Uma breve revisão teórica sinaliza a possibilidade da utilização de ferramentas quantitativas para análise de fenômenos sociais. São mencionados indices de centralidade e é utilizado o grau como base de análise.
\end{abstract}

Palavras-chave: exportação; consórcio; redes sociais; centralidade; grau.

\section{Introdução}

O Brasil, considerando o seu contexto exportador, ocupa uma posição pouco expressiva em relação ao cenário mundial, visto que o valor exportado pelo Brasil equivale a $1,08 \%$ do valor exportado mundialmente. Dentro desse contexto, a participação das empresas de pequeno e médio porte é baixa, pois estas são responsáveis por $2,03 \%$ do valor exportado, enquanto as grandes empresas são responsáveis por $89 \%$.

Especificamente sobre o setor de software, no ano 2000, o Brasil ocupava o sexto lugar como país exportador, dentro de uma lista de países que tinha os Estados Unidos em primeiro e a China em segundo lugar. Nesse mesmo ano, as exportações de software foram responsáveis por $1,5 \%$ do Produto Interno Bruto (PIB) brasileiro e pela geração de 158.000 empregos. A produção de software no Brasil é distribuída entre empresas de grande e pequeno e médio porte, sendo apenas $2 \%$ equivalentes ao número de grandes empresas.

Compreende-se que a formação em redes deve facilitar a inserção exportadora das pequenas e médias empresas, porque a rede permite que essas empresas realizem uma série de ações de difícil alcance caso agissem isoladamente. 
Para a realização deste estudo foi escolhido um consórcio de exportação de software, que é um tipo de organização em rede, situado em Campinas, no estado de São Paulo.

A metodologia utilizada consiste em uma pesquisa qualitativa realizada através de estudos de caso. Para a coleta de dados utilizou-se entrevista estruturada, aplicada às empresas e à coordenação do consórcio. Acredita-se que os resultados encontrados serão positivos em relação à inserção exportadora das empresas, graças aos aspectos relacionados à formação dessas empresas em rede.

\section{Redes Sociais e metodologia de análise}

Na concepção teórica de Powell \& Smih-Doerr (1994), redes sociais podem ser consideradas ferramentas de análise de relações sociais informais, ou como sendo estruturas de governança propriamente ditas. A utilização da perspectiva de redes sociais para a análise de estruturas de governança, trás à tona o aspecto relacional dos agentes envolvidos no sistema, sendo governança caracterizada por dinâmicas que ganham força própria para atuar em um objeto ou ação, cuja conduta criada por seus atores se faz seguida por todos os agentes relacionados.

Estruturas de governança, considerando a materialização do movimento de suas dinâmicas, atuam muitas vezes como mecanismos na constituição de arranjos organizacionais como clusters, arranjos produtivos locais (APLs) ou consórcios, formação privilegiada nesta pesquisa.

A análise das redes sociais deve considerar aspectos sob os quais ocorrem as trocas de informações, quais e porquê determinados agentes estão ligados, a força do vínculo entre eles (forte ou fraco) e a influência das relações na rede. Para tanto, aspectos como o tempo da ligação e fatores que envolvem confiança, passam a complementar esta análise. Ao mencionarmos as "trocas" ocorridas nas redes, entendemos que elas podem caracterizar troca de informações, de idéias, compartilhamento de ações etc, podendo ser a estrutura da rede uma grande fonte de oportunidades ou restrições.

Ao focarmos a relação entre os agentes, focamos na verdade a ligação entre eles, sendo que o tipo de relação é determinante para delinear a estrutura da rede. A definição de força da ligação foi desenvolvida por Granovetter (1973) como sendo "uma combinação, provavelmente linear, de tempo, intensidade emocional, intimidade (confidência mútua), e serviços recíprocos que caracteriza uma ligação", pressupondo que as ligações são positivas e simétricas e que cada fator é independente um do outro. Desta maneira, podem existir ligações fortes, fracas ou ausentes.

Segundo Dacin, Ventresca e Beal (1999), os trabalhos de Granovetter $(1985,1992)$ têm papel determinante no que tange a análise de redes no contexto social. Redes sociais traduzem para a estrutura das redes o papel das relações, como a geração de confiança para em contextos de relações econômicas, por exemplo (GRANOVETTER, 1985, 1992).

Apesar de vários métodos de análise terem sido desenvolvidos considerando atores, subgrupos de atores e grupos, na concepção da teoria de redes a unidade de análise é um conjunto de ligações formado a partir de um conjunto de atores. Quando medidas as variáveis estruturais de um único conjunto de atores, segundo Wasserman \& Faust (1994), temos uma rede uni-modal, sendo que na perspectiva de redes sociais os atores analisados são unimodais. 
Segundo Smolka (2006) citando Wasserman \& Faust (1994), há grande importância na menção das notações utilizadas para demonstrar as relações, sendo que as três principais utilizadas pela análise das redes sociais são: grafo-teóricas, sociométricas e algébricas. A notação sociométrica representa uma continuação da grafo-teórica (WASSERMAN \& FAUST, 1994), que é representada e utilizada neste trabalho.

O foco na força das ligações proposto por Granovetter (1973), seria suportado na notação sociométrica, que pode demonstrar relações com valores, mas não demonstrável na teoria dos grafos. Como neste trabalho apresentaremos o desenho das redes formado a partir das relações entre um conjunto de atores através de um grafo, os efeitos da governança e de fatores como confiança serão mencionados como parte de uma análise qualitativa, mas não como uma ferramenta para demonstrar as relações.

A apresentação dos dados se dá através de sociogramas das relações entre os atores na forma de matrizes. Ainda segundo Smolka (2006) citando Wasserman \& Faust (1994), as sociomatrizes são formadas por atores que enviam (as linhas) e atores que recebem (as colunas) as ligações. "A sociomatriz para uma relação binária é exatamente a matriz adjacente para o grafo que quantifica as ligações entre os atores da relação em questão" (WASSERMAN \& FAUST, 1994).

Este tipo de notação tem sido considerado suficiente como foco de análise nos estudos de redes, além de ser traduzível para softwares que podem fazer uma conversão dos dados em representações gráficas, como adotado para a apresentação dos resultados neste artigo.

Ainda quanto a sociomatriz, podem ser medidos índices de centralidade, que auxiliam na análise que considera conceitos da sociologia econômica como acesso e controle de recursos. Neste caso, os atores que se encontrarem em posições centrais na rede serão os responsáveis por maiores intermediações e terão maior acesso a recursos. A centralidade e o prestígio são então consideradas como classes do conceito de visibilidade de Knoke \& Burt citado por Wasserman \& Faust (1994), não explorado neste trabalho.

Dada a importância da centralidade para este trabalho, e na tentativa de melhor contextualizar a questão da centralidade, extraído de Freeman (1979), temos que a idéia de centralidade foi primeiramente introduzida nas discussões sobre comunicação humana por Bavelas em 1948. Sua preocupação era especialmente quanto à comunicação em pequenos grupos, sendo sua hipótese a relação de influência da centralidade estrutural em processos de grupos.

Vários estudos foram então produzidos, baseados na questão da resolução de problemas, no Laboratório de Redes do M.I.T., tendo sido conduzidos por Harold Leavitt (1949) e Sidney Smith (1950) e depois reportados por Bavelas (1950) e Bavelas e Barrett (1951) e descritos em detalhes por Leavitt (1951). A conclusão de todos os trabalhos corroboraram com a hipótese de que a eficiência dos grupos estava relacionada à centralidade na rede.

Ainda segundo Freeman (1979), outra aplicação importante da análise foi dada por Czepiel (1974), que utilizou o conceito para explicar padrões de difusão de inovação tecnológica na industria de aço.

Quanto aos índices, apesar de termos como possíveis índices de centralidade e prestígio o grau (degree), a proximidade (closeness), e a intermediação (betweenness), nesta pesquisa o grau será o índice de centralidade adotado para a análise das redes, correlacionando o desenho da rede com resultados de faturamento de exportações.

Segundo Wasserman \& Faust (1994), o nível de centralidade, medido pelo grau, determina a "localização da ação" na rede e o grau é a medida de atividade de um ator nesta rede. Além disto, "O grau é um índice importante para o potencial de atividade de comunicação do ator" 
podendo o ator exercer o papel de coordenador da rede de acordo com seu grau (FREEMAN, 1979, pg. 221). O grau é medido através do número de ligações que um ator recebe. Dada a sociomatriz de uma rede social $n$ e considerando $d\left(n_{i}\right)$ como sendo o número de ligações do ator $i$ desta rede, tem-se o índice grau de centralidade, $\mathrm{C}_{\mathrm{D}}\left(\mathrm{n}_{\mathrm{i}}\right)$, expresso na fórmula:

$$
C_{D}\left(n_{i}\right)=d\left(n_{i}\right)=x_{i+}=\sum_{j} x_{i j}=\sum_{j} x_{j i} .
$$

A aplicação desta fórmula, que resulta no grau de um ator na rede, é a base da análise dos dados deste trabalho, considerando que os dados de faturamento de exportações do Actminds farão parte da apresentação dos resultados.

\section{Quadro exportador brasileiro, a pequena e média empresa e consórcios de exportação}

A exportação é vista tradicionalmente como o primeiro passo para uma empresa entrar no mercado externo, sendo uma oportunidade interessante de ganhar experiência no mercado internacional (LU \& BEAMISH, 2001).

Embora seja a forma mais simples de entrada no mercado externo, não significa que seja fácil exportar. Leonidou (2004) aponta dificuldades que as pequenas e médias empresas podem enfrentar em relação à exportação: problemas em identificar, relacionar e contatar mercados externos; pessoal inadequado/não treinado; falta de capacidade de produção para exportação; adaptação do produto às exigências do mercado externo; não familiaridade com os procedimentos burocráticos de exportação; desconhecimento de diferentes hábitos e atitudes dos consumidores externos; dificuldade de divulgar seus produtos no exterior.

As empresas de pequeno e médio porte brasileiras, embora em relação a empresas exportadoras apresentem um número significativo, contribuem pouco em termos de valor exportado. Segundo dados do Sebrae (2004) a análise das exportações brasileiras por porte de empresa mostra que dos 20.902 estabelecimentos que exportaram em 2004, 4.957 (24\%) são micro empresas, $5.833(28 \%)$ são pequenas, 5.254 (25\%) são médias e $4.213(20 \%)$ são grandes. As 3\% restantes não foram classificadas. Em relação ao valor exportado, a representatividade das micro empresas em 2004 foi de $0,03 \%$, das pequenas de $2 \%$, das médias de $8 \%$ e das grandes empresas de $89 \%$ (Tabela 1 ).

Tabela 1: Número de empresas e valor exportado segundo o porte da firma - 2004

\begin{tabular}{lcccc}
\hline Porte da Empresa & $\begin{array}{c}\mathbf{N}^{\circ} \text { de empresas } \\
\text { exportadoras }\end{array}$ & $\mathbf{\%}$ & $\begin{array}{c}\text { Valor exportado (US\$ } \\
\text { milhões) }\end{array}$ & $\%$ \\
\hline Micro & 4957 & 24 & 302.138 .945 & 0,31 \\
Pequena & 5833 & 28 & 2.252 .835 .408 & 2,3 \\
Média & 5254 & 25 & 7.809 .633 .376 & 8,1 \\
Grande & 4213 & 20 & 85.880 .361 .068 & 89 \\
Não classificadas & 645 & 3 & 230.269 .545 & 0,2 \\
Total & 20902 & 100 & 96.475 .238 .342 & 99,91 \\
\hline Fonte: adaptado de Sebrae (2004) & & &
\end{tabular}

Estudos efetuados em vários países sobre a cooperação visando a exportação mostram que a formação de consórcios é um conceito promissor no engajamento das pequenas e médias empresas a operarem no mercado internacional. Os consórcios e pólos de exportação 
formados no Brasil, sobretudo a partir dos anos 90, foram criados com a idéia de potencializar a expansão dos mercados externos, o que acarretou na definição de estratégias de ações conjuntas entre as pequenas e médias empresas. Através do apoio da política nacional, estes consórcios foram beneficiados pelas ações de apoio em marketing e gestão tecnológica, o que tornou possível um maior engajamento conjunto das empresas na atividade de exportação (GUIMARÃES, 2002).

Mas, foi depois da criação da APEX em 1997, hoje denominada APEX Brasil, que a formação de consórcios de pequenas e médias empresas ganhou força, sendo que atualmente a maioria dos consórcios existentes no país foi criada com o apoio da APEX Brasil.

O consórcio de exportação pode ser caracterizado como um conjunto de empresas com interesses comuns que se agrupam e se organizam em torno de uma entidade estabelecida juridicamente, sem fins lucrativos, visando promover o acesso de seus produtos e serviços no mercado internacional. As empresas consorciadas buscam, a partir de objetivos comuns, a melhoria da oferta exportável e da promoção de exportações (SEBRAE, 2006).

Segundo Minervini (1997), as empresas que participam de um consórcio de exportação podem obter as seguintes vantagens: (1) diminuição da dependência de um número reduzido de fornecedores; (2) desenvolvimento de uma cultura exportadora e redução do custo de inexperiência, devido ao aprendizado conjunto; (3) obtenção de informações sobre os mercados internacionais a serem explorados para poder entrar nesses mercados com mais segurança; (4) aumento do poder de negociação com fornecedores, clientes, bancos e instituições governamentais; (5) alcance de economias de escala, reduzindo os custos unitários de produção e as despesas de exportação; (6) desenvolvimento de uma marca (do consórcio) mais forte; (7) aumento da lucratividade; (8) possibilidade de melhoria da forma de apresentação de embalagens e rótulos e (9) possibilidade de estabelecer parcerias internacionais.

\section{Setor de Tecnologia da Informação (TI) com foco em software}

No Brasil, o setor produtor de software ainda é pouco conhecido, sem estatísticas consistentes, devido, em boa medida, ao fato de ter um desenvolvimento recente, com maciça participação de pequenas empresas e com o mesmo elevado grau de segmentação verificado internacionalmente. Por outro lado, dada a sua elevada contribuição à modernização da sociedade, é um segmento que apresenta tendência de expressivo crescimento.

Até 2001, segundo dados levantados do catálogo SOFTEX (2002), o Brasil era o sétimo mercado de software do mundo, com taxa média de crescimento anual, desde 1995, na ordem de $11 \%$, sendo a maior do setor de TI, três vezes maior que a de hardware e aproximadamente cinco vezes maior que a do PIB nacional. Ainda tendo como fonte os dados do SOFTEX, podemos complementar mencionado que entre 1991 e 2001, o mercado de software brasileiro (desenvolvedoras de programas, processamento de dados e atividades de banco de dados) cresceu de 4,3 mil em 1994 para 5,4 mil em 200, em um total de 10,7 mil empresa com atividades potenciais de software. Vale resaltar que $82 \%$ destas empresas são micro empresas, $2 \%$ grandes e, no total delas, 2.398 são desenvolvedoras de software.

A escolha do setor de TI, tem sua base principal no dado proveniente da Pesquisa Anual de Serviços do ano 2000 (PAS/2000), realizada pelo IBGE, que indica que 98,4\% das 15 mil empresas de informática existentes no Brasil possuíam menos de 50 funcionários. Estas empresas faturaram, juntas, quase $\mathrm{R} \$ 2$ bilhões naquele ano. Segundo o mesmo estudo, as 
empresas de telecomunicações somaram 1,4 mil, das quais 91,7\% possuíam até 50 funcionários. A receita destas empresas somadas chegou a R $\$ 580$ milhões.

\section{Actminds}

O Actminds possui características de consórcio, mas não tem constituição jurídica, apresentando-se no exterior como joint venture. As primeiras elucubrações sobre a formação deste consórcio se deram através de representantes de empresas de TI que participavam das reuniões e assessoria do SOFTEX, fomentados pelo então coordenador do núcleo de Campinas, hoje consultor responsável por relações do Actminds. Formalmente o agrupamento das empresas e as reuniões periódicas foram iniciados em 2004.

Há um conjunto de onze empresas (pequenas e médias) que fazem parte do consórcio, sendo que apenas nove delas apresentam-se "ativas" (sendo a inatividade uma condição acordada pelos membros do consórcio, podendo esta ser revertida à atividade segundo alguns critérios não explicitados neste artigo).

Com relação a operação do consórcio, há um estatuto e um modelo de gestão e operação que determinam as ações das empresas e da coordenação segundo uma configuração que mantém um conselho composto por representantes das empresas do consórcio, comitês administrativo, financeiro jurídico, de vendas e marketing, comitês verticais de mercado - responsáveis pela administração das estratégias comerciais nos mercados-alvo -, e a coordenação. As reuniões acontecem semanalmente e as empresas que compõe o consórcio devem estar até $50 \mathrm{~km}$ de distância de Campinas. O órgão de fomento que subsidia as exportações do Actminds é a APEX e a relação de subsídio é de $50 \%$ ou igual contrapartida.

A figura 1 apresenta dados consolidados de faturamento de exportações do consórcio, não por empresa isolada, entre os anos de 1998 e 2005, novamente mencionando que o Actminds se formou a partir de 2004. Os dados de 2006 e 2007 esboçam uma previsão de faturamento, considerando a tendência de ascensão da curva.

Figura 1. Dados consolidados do faturamento do Actminds

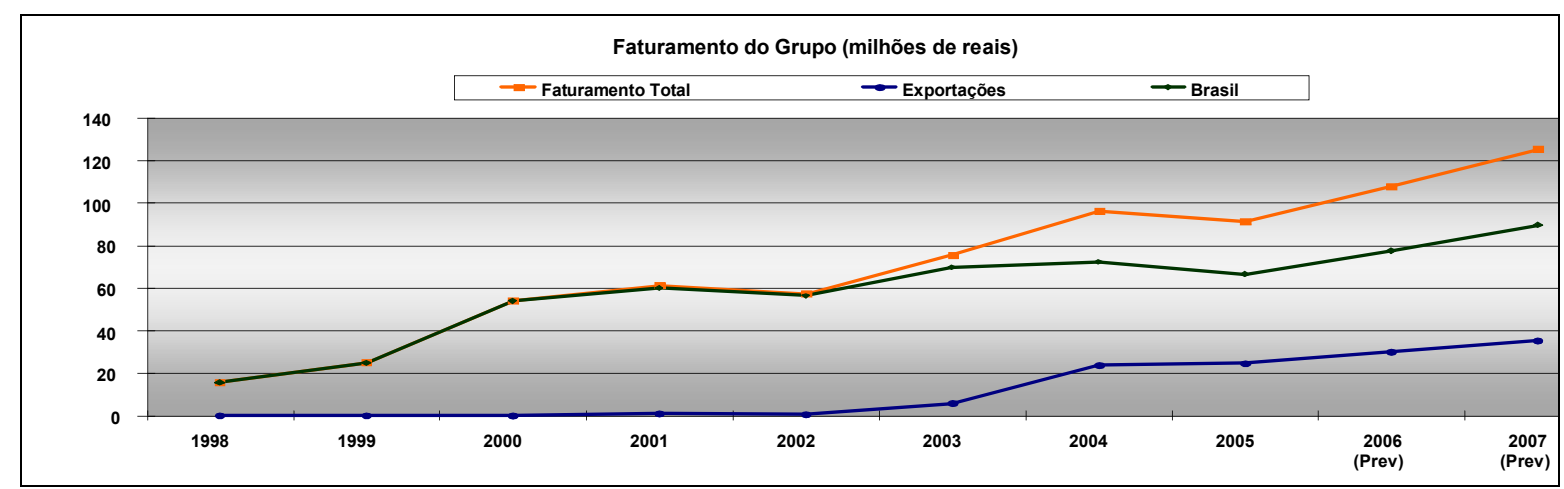

Fonte: Relatório Anual Actminds 2005 (adaptado) 


\section{Metodologia de coleta de dados}

A metodologia empregada foi a da pesquisa qualitativa, através de estudo de caso em um consorcio de exportação localizado em Campinas-SP, chamado Actminds. Das nove empresas ativas, entrevistou-se seis delas e a coordenação do consórcio. As entrevistas foram presenciais, seguiram um roteiro estruturado e foram gravadas.

Os profissionais entrevistados foram os efetivos representantes de cada empresa no Actminds e os nomes das empresas que compõe o consórcio são: Matera, Programmers, FITEC, Progonos, Tele Design, IPS, SER, Blue Pex, CI\&T, Grupo Net e HST.

\section{Resultados}

Na figura 2, temos o desenho das empresas que compõe o Actminds que exportaram antes da formação do consórcio, deixando claro que não havia ligação entre os atores nesta rede. Já na figura 3, o grafo representa o desenho da rede de exportação de software formada depois da organização das empresas em consórcio.

Figura 2. Exportação antes do Actminds

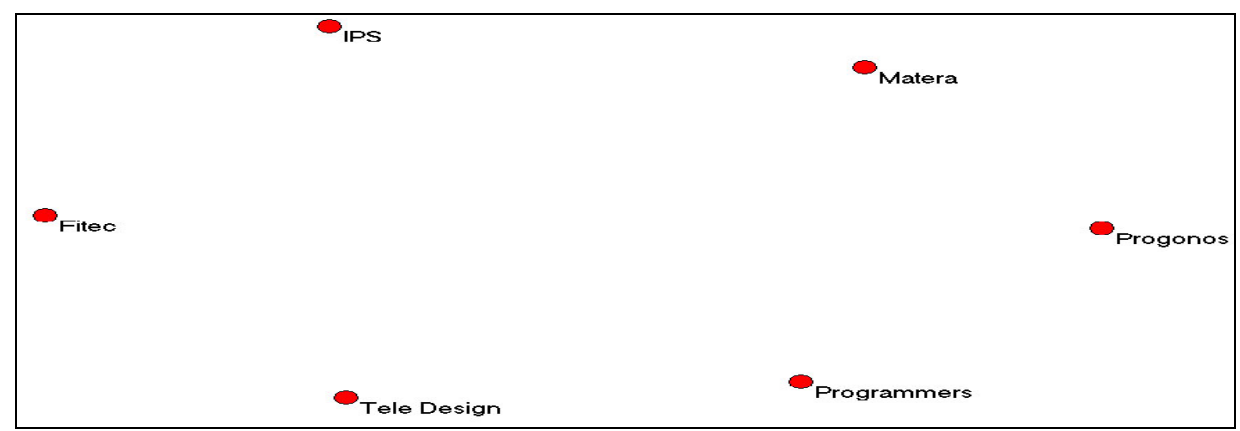

Figura 3. Redes exportação depois do Actminds

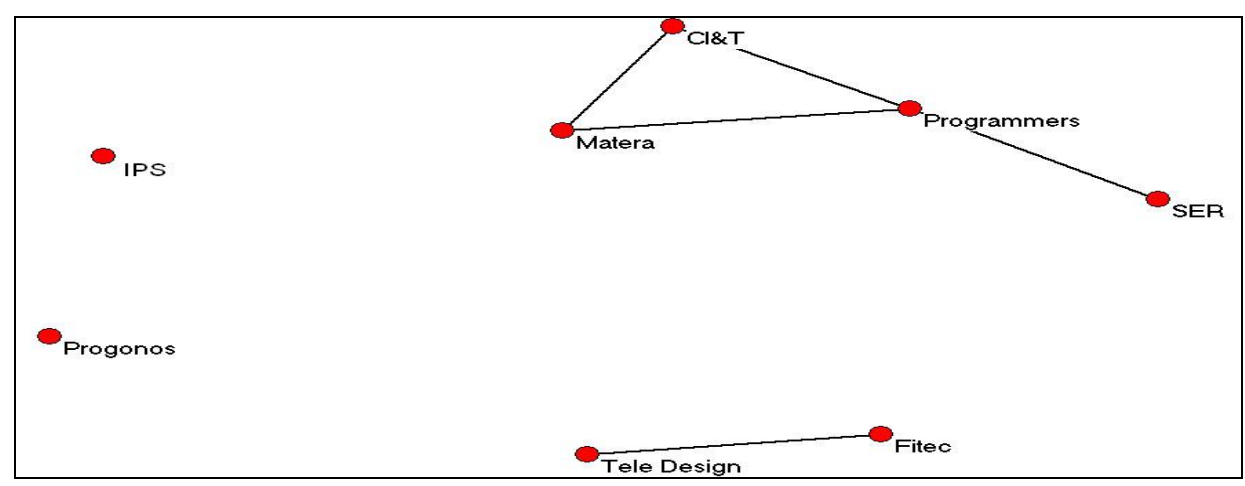

Com base na tabela 2, podemos verificar o grau dos atores envolvidos na rede que compõe o Actminds, calculado através da fórmula $C_{D}\left(n_{i}\right)=d\left(n_{i}\right)=x_{i+}=\sum_{j} x_{i j}=\sum_{j} x_{j i}$. A

Programmers é a empresa com maior grau, tendo expressa ligação com outras três empresas da rede, seguida pela Matera e Cl\&T, tendo ambas ligação com outras duas empresas da rede. 
Tabela 2. Grau de Centralidade e faturamento de exportações

\begin{tabular}{lcc}
\hline \multicolumn{1}{c}{ Atores } & $\begin{array}{c}\text { Grau de } \\
\text { centralidade }\left(\mathbf{C}_{\mathbf{D}}\right)\end{array}$ & $\begin{array}{c}\text { Faturamento de } \\
\text { exportação }\end{array}$ \\
\hline Programmers & 3 & $\mathrm{R} \$ 3,5$ milhões \\
Matera & 2 & $\mathrm{R} \$ 1,5$ milhões \\
CI\&T & 2 & $\mathrm{R} \$ 1,2$ milhão \\
Fitec & 1 & $\mathrm{R} \$ 18,9$ milhões \\
Tele Design & 1 & $\mathrm{R} \$ 0,00$ \\
SER & 1 & $\mathrm{R} \$ 0,00$ \\
Progonos & 0 & $\mathrm{R} \$ 0,00$ \\
IPS & 0 & $\mathrm{R} \$ 0,00$ \\
\hline Fons
\end{tabular}

Fonte: Entrevistas com as empresas

A notação do grau é evidenciada nos grafos, sendo que podemos ainda fazer uma correlação simples entre o maior grau nas empresas que são maiores, portanto têm mais condições financeiras para utilizar os subsídios da Apex, já que é exigida uma contrapartida de $50 \%$ dos investimentos. Outra correlação possível é que as empresas que são mais centrais na rede, ou seja, que possuem maior número de ligações, são também àquelas que possuem maior faturamento de exportações.

A Fitec em especial é uma das maiores empresas do grupo e também a de maior faturamento, apesar de possuir grau igual a 1, inferior a Programmers, Matera e Cl\&T. Este dado pode ser atribuído ao fato de que a Fitec possui um forte histórico de exportações. A Lucent Technologies adquiriu as empresas Zetax e Batic e então passou a exportar, através da Fundação, para principalmente os EUA, tendo em 2001 atingido um faturamento de R\$ 23 milhões em exportações. Desta forma, considera-se a Fitec uma exceção para a correlação entre o grau e o faturamento de exportação das empresas na rede do Actminds.

Apesar da SER e da Tele Design terem faturamento de exportações igual a zero, estão ligadas a outros atores da rede por estarem engajadas em parcerias com perspectiva de projetos, sendo o faturamento atrelado à consolidação destes negócios. Assim, entendemos que em momento posterior teremos possivelmente faturamento de exportações advindo destas empresas, mas com valores que não devem superar os valores atingidos pelas empresas que já têm faturamento de exportações.

A Progonos e IPS, ainda não exportaram e nem estabeleceram parcerias para exportação desde a formação de Actminds.

Mesmo não sendo dados passíveis de serem analisados quantitativamente, todas as empresas entrevistadas, relataram os benefícios de fazerem parte do consórcio, destacando: acesso a oportunidades, riscos e os custos de explorar novas oportunidades de mercado assumidos conjuntamente, a permuta de competências e utilização de know-how de outras empresas, a possibilidade de oferecer uma linha de produtos de qualidade superior e mais diversificada, a condição de exercer uma pressão maior no mercado aumentando a força competitiva através de uma marca única e compartilhamento de recursos, como atrelar ao consórcio o maior nível de certificação atingido por determinada empresa.

O fator confiança, também surge de maneira muito proeminente nos relatos dos representantes das empresas do Actminds, que atribuem ao fato de "confiarem" uns nos outros, a predileção pelos membros do consórcio no estabelecimento de parcerias comerciais locais, apesar de o consórcio visar as exportações, e compartilhamento de informações tecnológicas e de mercado. 
A dinâmica de operações do consórcio parece outro fator a contribuir para que a rede de fato traga benefícios para seus atores. As reuniões semanais mantêm as empresas em permanente contato (freqüência) e o requisito geográfico (localização), que prima por empresas que estejam próximas em um raio de até $50 \mathrm{~km}$, facilita o acesso às reuniões e às dependências de cada empresa quando necessário.

\title{
8. Conclusão
}

Os resultados da pesquisa corroboram com a hipótese inicial de que a formação em rede facilita a exportação de software de pequenas e médias empresas, e os dados de evolução de faturamento do grupo evidenciam ainda mais os aspectos positivos deste agrupamento.

O consórcio, como sendo uma estrutura de governança, possibilita de fato que a conduta criada por seus atores seja seguida por todos os agentes relacionados, o que se verifica facilmente no engajamento das empresas nas estratégias delineadas pelo consórcio, além de manterem fidelidade às regras do estatuto que rege as ações do grupo.

A correlação entre o grau e o faturamento de exportações nos permite evidenciar a possibilidade de uma análise quantitativa frente à perspectiva de redes sociais utilizando uma notação grafo-teórica, sendo que a notação sociométrica representa uma continuação da grafoteórica, suportando análises qualitativas como a força dos vínculos.

\begin{abstract}
This paper has the objective of verifying if the consortium organization makes easier the export insertion of small and medium Brazilian companies from the software industry. The analyses is performed under the social networks perspective and conclusions are based on the qualitative research which uses a case of study considering the Actminds, a software export consortium located in Campinas - SP. A brief theoretical review mentions the possibility of using quantitative tools to analyze social phenomena. Centrality indexes are mentioned and the degree is used as analyses basis.
\end{abstract}

Key-Words: export; consortium: social networks; centrality; degree.

\section{Referências}

BAVELAS, A. A mathematical model for group structures. Human Organization, v 7, p.16-30, 1948.

BAVELAS, A. Communication patterns in task oriented groups. Journal of the Acoustical Society of America, v 22, n 3, p. 221 e p. 271-282, 1950.

BAVELAS, A. e Barret, D. An experimental approach to organizational communication. Personnel. Cap. 27, p.366-371, 1951 .

DACIN, M. Tina; VENTRESCA, Marc J. e BEAL, Brent D. The embeddedness of organizations: dialogue and directions. Journal of Management, v 25, n. 3, p. 317-356, 1999. 
FREEMAN, L.C. Centrality in social networks: Conceptual clarification. Social Networks. v. 1, p. 215-239, 1979.

cross'

GRANOVETTER, Mark S. The strength of weak ties. American Journal of Sociology, v.78, n. 6, May 1973.

GRANOVETTER, Mark. Economic action and social structure: the problem of embeddedness. American Journal of Sociology. Chicago, v. 91, n.3, p. 481-510, 1985.

GRANOVETTER, Mark. Problems of explanation in economic sociology. IN: NOHRIA, Nitin e ECCLES, Robert G. (Org.), Networks and organizations: structure, form, and action. Boston: Harvard Business School Press, Cap. 1, p. 25-5, 1992.

GUIMARÃES, E. P. Política de Exportação Brasileira para Pequenas e Médias Empresas. In: BNDES: O Desafio das Exportações, Ed. BNDES, Rio de Janeiro, 2002.

CZEPIEL, J. A. Word of mouth processes in the diffusion of a major technological innovation. Journal Of Marketing Research, v.11, p.172-180, 1974.

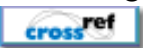

LEAVITT, H. J. Some effects of communication patterns on group performance. Journal of Abnormal and Social Psychology, v. 46, n. 1, p. 38-50, 1951.

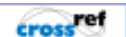

LU, J. W \& BEAMISH, P. W. The internationalization and performance of SMEs. Strategic Management Journal.n. 22, p. 565-586, 2001.

cross ref

MINERVINI, N. Exportar: competitividade e internacionalização. Makron Books, São Paulo, 1997.

$\begin{array}{lllll}\text { PESQUISA ANUAL } & \text { DE } & \text { SERVIÇOS. }\end{array}$ http://www.ibge.net/home/estatistica/economia/comercioeservico/pas/analisepas2000.shtm (consultado em 20/05/05).

POWELL, WW. \& SMITH-DOERR, L.: "Networks and Economic Life". In: SMELSER, N.J. \& SWEDBERG, R. (eds.) Handbook of EconomicSociology. Princeton, Russell Sage Fundation, p.368-402, 1994.

SEBRAE. Desempenho exportador das micro e pequenas empresas brasileiras. Boletim. Primeiro semestre de 2004. Versão final, 2004.

SEBRAE. http://www.sebraema.com.br/produto/consorcio.htm (consultado em 22/02/2006)

SMITH, S. L. Communication Pattern and the Adaptability of Task-oriented Groups: an Experimental Study. Cambridge, MA: Group Networks Laboratory, Research Laboratory of Electronics. Massachucetts Institute of Technology, 1950.

SMOLKA, R. B. Redes de cooperação entre EBT'S do setor médico-hospitalar da região de São Carlos, Araraquara e Ribeirão Preto. 2006. 166 f. Dissertação (Mestrado em Gestão de Produção) - Centro de Ciências Exatas e de Tecnologia, Universidade Federal de São Carlos, São Carlos. Mimeo.

SOFTEX. A indústria de software no Brasil 2002 - fortalecendo a economia do conhecimento - Projeto do Massachussets Institute of Technology. Campinas, 2002, 80 p.

WASSERMAN, S.; FAUST, K. Social network Analysis: methods and applications. Cambridge: Cambridge University Press, 1994.

\section{Dados dos autores}

Nome completo: Ana Paula de Lacerda Zacharias

Filiação institucional: Universidade Federal de São Carlos

Departamento: Departamento de Engenharia de Produção 
Endereço: R. Hermantino Coelho, 195 ap 43 - ed. Plaza. Mansões Sto Antônio - Campinas SP - Brasil. Cep: 13087-500

Telefones para contato: ++ 551932376567 ++ 551997257871

e-mail: anapaula@huc.com.br

Nome completo: Márcia Freire de Oliveira

Filiação institucional: Universidade Federal de São Carlos

Departamento: Departamento de Engenharia de Produção

Endereço: Av. Mato Grosso, 653 ap. 62 - Zona 2. Cianorte -PR - Brasil. Cep: 87200-000

Telefones para contato: ++ 554436299939

e-mail: marciaf@dep.ufscar.br

Nome completo: Mauro Rocha Côrtes

Filiação institucional: Universidade Federal de São Carlos

Departamento: Departamento de Engenharia de Produção

Função ou cargo ocupado: Professor Adjunto

Endereço: Rua 13 de maio, 3665, Vila Nery - São Carlos - SP - Brasil. Cep: 13.569-040

Telefones para contato: ++551633518236 ext. 9225

e-mail:Mauro@dep.ufscar.br

Recebido para publicação em: 30/10/2006

Aceito para publicação em: 05/02/2007 